\title{
Modeling Responses to Peripheral Nerve Stimulation in the Dorsal Horn
}

\author{
Christine Beauchene ${ }^{1}$, Pierre Sacré ${ }^{2}$, Fei Yang ${ }^{3}$, Yun Guan ${ }^{3}$, and Sridevi V. Sarma ${ }^{1}$
}

\begin{abstract}
Pain is a protective physiological system essential for survival. However, it can malfunction and create a debilitating disease known as chronic pain (CP). CP is primarily treated with drugs that can have negative side effects (e.g., opioid addiction), and lose efficacy after long-term use. Electrical stimulation of the spinal cord or peripheral nerves is an alternative therapy that has great potential to reduce the need for drugs and has fewer negative side effects; but has been associated with suboptimal efficacy because its modulation mechanisms are unknown. Critical to advancing $\mathrm{CP}$ treatment is a deeper understanding of how pain is processed in the superficial and deep layers of the dorsal horn (DH), which is the first central relay station for pain processing in the spinal cord. Mechanistic models of the DH have been developed to investigate modulation mechanisms but are non-linear and high-dimensional and thus difficult to analyze. In this paper, we construct a tractable computational model of the $\mathrm{DH}$ in rats from LFP recordings of the superficial layer network and spiking activity of WDR neurons in the deep layer. By combining a deterministic linear time-invariant model with a stochastic point process model, we can accurately predict responses of the DH circuit to electrical stimulation of the peripheral nerve. The model is computationally efficient, low-dimensional, and able to capture the stochastic nature of neuronal dynamics in the DH; and is a first step in developing new therapies for CP.
\end{abstract}

\section{INTRODUCTION}

Acute pain is an early-warning physiological signal triggered in the nervous system, which is essential for survival. However, pain processing is fragile since inflammation, injury, and nervous system malfunction may divert its function, creating a debilitating disease known as chronic pain (CP). $\mathrm{CP}$ affects about 100 million adults in the US, with $\$ 600$ billion in annual medical expenses and lost productivity [1].

The primary treatment for $\mathrm{CP}$ is drugs, which have negative side effects (e.g., opioid addition), and lose efficacy after long-term use [2]. Alternatively, electrical stimulation of the spinal cord or peripheral nerves is associated with significantly less negative side effects, but successful outcomes are limited due to the poor understanding of its mechanisms [3].

To improve neurostimulation pain therapies, a critical but complicated question is "how does the dorsal horn process pain?" This remains an open question because the pain system is difficult to probe (experimental barriers) and difficult to analyze (computational barriers). External sensory inputs -

\footnotetext{
* This work is funded by the NIH/NINDS T32 NSO70201 to CB and NIH/NCCIH R01AT009401 to SVS and YG.

${ }^{1}$ Christine Beauchene and Sridevi V. Sarma are with the Institute for Computational Medicine, Biomedical Engineering, Johns Hopkins University, Baltimore, MD, USA. (corresponding author: cbeauch2@jhu.edu)

2 Pierre Sacré is with the Department of Electrical Engineering and Computer Science, University of Liège, Belgium.

3 Fei Yang and Yun Guan are with the Department of Anesthesia and Critical Care Medicine, Johns Hopkins Hospital, Baltimore, MD, USA.
}

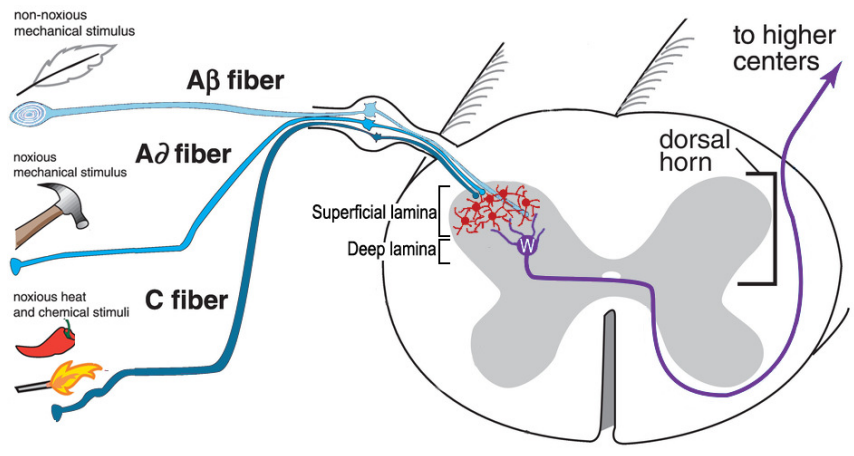

Fig. 1. An illustration of the dorsal horn in the spinal cord. Afferent fibers enter the superficial lamina and the WDR neuron (W) in the deep lamina projects the impulse to the brain.

noxious and innocuous - are processed first in the superficial and then deep layers of the dorsal horn (DH). The superficial layers (lamina I-II) contain inhibitory and excitatory neurons, which receive and integrate sensory inputs primarily from the $\mathrm{A} \delta$ and $\mathrm{C}$ afferent peripheral nerve fiber (Figure 1).

The deep layers (lamina III-V) consist of convergent neurons that receive noxious ( $\mathrm{A} \delta$ and $\mathrm{C}$ fibers) and innocuous (A $\beta$ fibers) inputs, and thus are also known as widedynamic-range (WDR) neurons. The superficial and deep layers form a modulatory circuit that partially regulates the ascending pain transmission through the centrally located WDR neurons. Electrophysiological recordings of these neurons provide important dynamic information about changes in neurophysiological responses to disease, injury, neurostimulation, or drug treatment for CP. However, differentiating subsets of DH neurons while recording the dynamics is challenging because the neuron's neurochemical identity is unknown prior to recording since tracing the staining of injected dye is complicated and inefficient.

A complement to conducting challenging biological experiments is to construct realistic mathematical models of the DH circuit. In 1965, Melzack and Wall proposed the first static model of pain modulation in the DH, known as the "gate-control theory" of pain [3]. It describes how DH inhibitory interneurons act as a functional gate that "opens" or "closes", thereby relaying or blocking, respectively, pain transmission to the brain. This model was timely as it showed the need for models, however, gate control theory fails to explain the dynamics in firing patterns that emerge under certain conditions (e.g. wind up, endogenous bursting) and the relationships between these patterns and pain conditions.

Since then, various detailed conductance-based dynamical models of inhibitory, excitatory, and WDR neurons in the DH and interconnections between these neurons have been built [4], [5], [6]. These models can reproduce some observed 
behaviors, but they assume a fixed circuit topology, are highdimensional, and nonlinear. Therefore, they are not amenable to analysis, i.e., analytically characterizing a set of sensory stimuli, model parameters, and treatment parameters that produce the observed firing patterns it is nearly impossible.

In this paper, we construct, for the first time, a tractable computational model of the $\mathrm{DH}$ circuit by combining deterministic and stochastic models. The model is constructed from a novel set of experimental data from the $\mathrm{DH}$ in rats generated using state-of-the-art electrophysiological techniques. Our results show that the dynamics of the local field potentials (LFP) from the superficial layers can be correctly estimated with a linear time-invariant (LTI) transfer function model and spiking activity of WDR neurons can be accurately predicted using a point process model.

\section{METHODS}

\section{A. Rodent Electrophysiology Recordings}

Electrophysiological measurements are recorded in vivo from four healthy adult male rats (all procedures approved by the Johns Hopkins University Animal Care and Use Committee). Fine-tip microelectrodes are used to measure neuronal activity in the superficial lamina and the deep lamina of the DH, which correspond to the LFPs and spiking activity from the WDR neurons, respectively (Figure 2a). The sampling frequency of the dataset is $500 \mathrm{~Hz}$. To perturb the $\mathrm{DH}$, a pulse input is applied to the peripheral sciatic nerve. Each pulse is $5 \mathrm{~mA}$, biphasic, and $0.5 \mathrm{~ms}$ long. For the LFP recordings, a paired-pulse input is applied using the same parameters with a $400 \mathrm{~ms}$ delay between the $1^{\text {st }}$ and $2^{\text {nd }}$ pulse. In total, ten trials are recorded for each animal. Due to the difficulties of simultaneously measuring from various subsets of DH neurons, the LFP and WDR responses are recorded individually. Thus, each of the trials is time-locked to the first pulse input for model construction (Figure 2b).

\section{B. Dorsal Horn Model}

The objective of the proposed $\mathrm{DH}$ model is to predict the superficial and deep lamina responses from only the stimulation pulse input. The LFP and WDR responses are modeled using a $2^{\text {nd }}$ order LTI discrete-time transfer function and a point process model, respectively. Figure $2 \mathrm{c}$ shows the



Fig. 2. a) A biophysical representation of the DH. The grey triangles represent the recording microelectrodes. The WDR neuron $(\mathrm{W})$ projects the impulse to the brain. b) Ten trials of LFP and WDR recordings time-locked to the input. c) A generalized block diagram representation of the $\mathrm{DH}$. generalized block diagram of the full model. To predict more accurate responses, the biphasic pulse input is transformed into a parameterized pulse input, $u(t)$, which is described in Section II-C. We delay $u(t)$ by $\sigma_{\mathrm{A}}$ and $\sigma_{\mathrm{C}}$ seconds before it enters the multi-input, single-output transfer function. The two transfer functions represent the $\mathrm{A} \beta$ fiber, $x_{\mathrm{A}}(t)$, and $\mathrm{C}$ fiber, $x_{\mathrm{C}}(t)$, components of the LFP response. Then, $u(t)$, $x_{\mathrm{C}}(t)$, and $x_{\mathrm{A}}(t)$ are incorporated into the point process model to estimate the WDR spiking activity.

\section{Discrete-Time Transfer Function Estimation and Analysis}

For each of the four rats, $70 \%$ of the LFP data are used to fit the $2^{\text {nd }}$ order LTI discrete-time transfer function. To identify the best fits, five parameters are optimized over a grid search listed in Table I. The parameterized input (Figure 3 ) is defined by three parameters

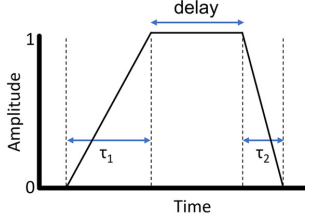

Fig. 3. Parameterized input representation of the pulse. $\left(\tau_{1}, \tau_{2}\right.$, and delay), and is delayed by $\sigma_{\mathrm{A}}$ and $\sigma_{\mathrm{C}}$ seconds.

TABLE I

PARAMETER SEARCH GRID

\begin{tabular}{|c|c||c|c|}
\hline$\tau_{1}, \tau_{2}$ & $\{0.1 \mathrm{~ms}, 0.9 \mathrm{~ms}, 0.19 \mathrm{~ms}\}$ & $\sigma_{\mathrm{A}}$ & $\{0.05 \mathrm{~ms}, 0.13 \mathrm{~ms}, 0.2 \mathrm{~ms}\}$ \\
\hline delay & $\{1 \mathrm{~ms}, 2 \mathrm{~ms}, 3 \mathrm{~ms}, 4 \mathrm{~ms}\}$ & $\sigma_{\mathrm{C}}$ & $\{130 \mathrm{~ms}, 160 \mathrm{~ms}, 190 \mathrm{~ms}\}$ \\
\hline
\end{tabular}

For each parameter combination, the coefficients of the transfer function listed in (1) are fit using the tfest Matlab command. The root-mean-squared error (RMSE) is computed between the last $30 \%$ of the recorded LFP data and the predicted LFP response using the fitted transfer function. For each rat, the final parameters are chosen such that the RMSE is minimized over all parameter combinations.

$$
\begin{aligned}
& \frac{X_{\mathrm{A}}(z)}{U(z)}=z^{-\sigma_{\mathrm{A}}} \frac{a_{0_{\mathrm{A}}}+a_{1_{\mathrm{A}}} z^{-1}}{1+b_{1_{\mathrm{A}}} z^{-1}+b_{2_{\mathrm{A}}} z^{-2}} \\
& \frac{X_{\mathrm{C}}(z)}{U(z)}=z^{-\sigma_{\mathrm{C}}} \frac{a_{0_{\mathrm{C}}}+a_{1_{\mathrm{C}}} z^{-1}}{1+b_{1_{\mathrm{C}}} z^{-1}+b_{2_{\mathrm{C}}} z^{-2}}
\end{aligned}
$$

\section{Point Process Model Estimation and Analysis}

We formulate a point process model to relate the spiking propensity of each WDR neuron to factors associated with the LFP response and features of the neuron's spiking history. A point process is a series of $0-1$ random events that occur in continuous time. For a neural spike train, the $1-\mathrm{s}$ are spike time events and the 0-s are the times at which no spikes occur. Neural spiking activity can be defined using a point process model in which we consider an observation interval $(0, T]$, and count the number of spikes, $N(t)$, in the interval $(0, t]$ for $t \in(0, T]$. A point process model of a neural spike train can be completely characterized by its conditional intensity function (CIF), $\lambda\left(t \mid H_{t}\right)$, which is defined as:

$$
\lambda\left(t \mid H_{t}\right)=\lim _{\Delta \rightarrow \infty} \frac{P\left(N(t+\Delta)-N(t)=1 \mid H_{t}\right)}{\Delta},
$$

where $H_{t}$ denotes the spike history up to time $t$. It follows from (2) that the probability of a single spike in a small interval $(t, t+\Delta]$ is approximately

$$
\operatorname{Pr}\left(\text { spike in }(t, t+\Delta] \mid H_{t}\right)=\lambda\left(t \mid H_{t}\right) \Delta .
$$


Details can be found in [7], [8]. When $\Delta$ is small, (3) is approximately the spiking propensity at time $t$.

The CIF generalizes the rate function of a Poisson process to a rate function that is history dependent. A spike train can be completely characterized by a CIF. Therefore, if we define a model for the CIF, then, as a result, we define a model for the spike train [9], [10]. For these analyses, we use generalized linear models (GLM) [11] to fit each CIF estimate framework to conduct statistical inferences [12].

Specifically, for each WDR neuron, the CIF is modeled as a function of the input pulse, the LFP response in the superficial layers, and the spiking history of the WDR neuron. For each neuron, the CIF has a multiplicative structure [13]:

$$
\lambda\left(t \mid H_{t}, x_{\mathrm{A}}, x_{\mathrm{C}}, u, \Theta\right)=e^{\alpha t} \lambda^{x}\left(t \mid \gamma, x_{\mathrm{A}}, x_{\mathrm{C}}, u\right) \lambda^{0}\left(t \mid H_{t}^{0}, \beta\right),
$$

where $\alpha$ is a constant history-independent term, $\lambda^{x}$ is the effect of the superficial lamina, and $\lambda^{0}$ describes the effect of the WDR neuron's own spike history $\left(H_{t}^{0}\right)$ on the neural response. The parameter vector $\Theta \equiv[\alpha, \gamma, \beta]$ is estimated from the data. Both $\lambda^{x}$ and $\lambda^{0}$ are dimensionless.

The full CIF model (4) is composed of distinct CIFs for each different kind of covariates, in order to assess how much each component contributes to the spiking propensity of the neuron. The superficial layer CIF, $\lambda^{x}$, is given by

$$
\lambda^{x}\left(t \mid \gamma, x_{\mathrm{A}}, x_{\mathrm{C}}, u\right)=\exp \left\{\beta_{u} u(t)+\beta_{\mathrm{A}} x_{\mathrm{A}}(t)+\beta_{\mathrm{C}} x_{\mathrm{C}}(t)\right\},
$$

where $\gamma=\left[\beta_{u}, \beta_{\mathrm{A}}, \beta_{\mathrm{C}}\right], u(t)$ is the parameterized input, $x_{\mathrm{A}}(t)$ is the $\mathrm{A} \beta$ fiber response, and $x_{\mathrm{C}}(t)$ is the $\mathrm{C}$ fiber response. The WDR spiking history CIF, $\lambda^{0}$, is defined as

$$
\begin{aligned}
\lambda^{0}\left(t \mid H_{t}^{0}, \beta\right)= & \exp \left\{\sum_{i=1}^{12} \beta_{i} N(t-i+1: t-i)\right. \\
& +\beta_{13} \sum N(t-63: t-13) \\
& \left.+\beta_{14} \sum N(t-980: t-750)\right\},
\end{aligned}
$$

where $\beta=\left\{\beta_{i}\right\}_{i=1}^{14}$ and $N(a: b)$ is the number of spikes observed in the time interval $[a, b)$. For all parameters, the chosen bin length is $0.02 \mathrm{~ms}$. The short-time (ST) history parameters, $\left\{\beta_{i}\right\}_{i=1}^{12}$, measure the effects of the spiking history in the previous $0.24 \mathrm{~ms}$ and therefore can capture refractoriness and/or bursting on the spiking probability. The summed short-time (SST) history parameter, $\beta_{13}$, and the summed long-time (SLT) history parameter, $\beta_{14}$, instead, measure the effects of the spike history from $0.26 \mathrm{~ms}$ to 1.26 $\mathrm{ms}$ and from $15 \mathrm{~ms}$ to $19.6 \mathrm{~ms}$ prior the time $t$, respectively. Because of its optimality properties, we choose a likelihood approach for fitting and analyzing the parametric model of the CIF. The parameters are efficiently computed using the iterative reweighted least squares algorithm.

In order to determine which factors are necessary for accurate spiking prediction, we compare three different point process models: M1 $\left(\lambda=e^{\alpha t} \lambda^{x}\right), \mathrm{M} 2\left(\lambda=e^{\alpha t} \lambda^{0}\right)$, and M3, which is the full model described in (4). To evaluate the predictive power of the point process models, we employ a receiver operating characteristic (ROC) curve analysis. To compute the ROC curve, the $\lambda$ for each model is thresholded from 0 to 1 by steps of 0.001 . At each threshold step, for each point that $\lambda$ is above the threshold it is considered a spike, while everything below it is not. The computed truepositive rate and false-positive rate create the ROC curve.

The time windows for the ST, SST, and SLT covariates (i.e $N(t-63: t-13)$ for the SST) are chosen such that the area under the ROC curves (AUC) is optimized over a search grid. The search values for the time windows are ST (2 time bins to 30 time bins), SST (2 time bins to 70 time bins starting after the current ST value), SLT (500 time bins to 1000 time bins). The combination of time windows listed in (6) maximize the AUC values over all four rats.

Leave-one-out cross-validation is used to evaluate the performance of each point process model. For each of the ten folds, nine trials are used for training and a different single trial left out to be used for validation. For each rat, a oneway ANOVA is applied to determine if significant differences exist between the AUC values for each model. The Tukey HSD test evaluates the pairwise comparisons $(\alpha=0.05)$.

\section{RESULTS}

\section{A. Predicting the Superficial Lamina LFP Response}

A $2^{\text {nd }}$ order LTI discrete-time transfer function is used to capture the dynamics of the superficial lamina in response to the paired-pulse parameterized input. Figure 4 shows a comparison of all ten recorded LFP trials (displayed in black) and the optimized transfer function output (shown in red) for each rat subject (S). The RMSE between the recorded and estimated LFP responses are 0.142, 0.058, 0.116, 0.162, respectively. Tables II and III list the optimal values for the parameterized input and the corresponding fitted transfer function coefficients, respectively. Therefore, using an LTI system can quickly and accurately predict the LFP response to a paired-pulse input.

TABLE II

THE OPTIMAL PARAMETERS FROM THE GRID SEARCH.

\begin{tabular}{|c|c|c|c|c|c|}
\hline $\mathrm{S}$ & $\tau_{1}$ & $\tau_{2}$ & delay & $\sigma_{\mathrm{A}}$ & $\sigma_{\mathrm{A}}$ \\
\hline 1 & $0.19 \mathrm{~ms}$ & $0.09 \mathrm{~ms}$ & $3 \mathrm{~ms}$ & $0.2 \mathrm{~ms}$ & $190 \mathrm{~ms}$ \\
\hline 2 & $0.19 \mathrm{~ms}$ & $0.09 \mathrm{~ms}$ & $3 \mathrm{~ms}$ & $0.2 \mathrm{~ms}$ & $190 \mathrm{~ms}$ \\
\hline 3 & $0.19 \mathrm{~ms}$ & $0.01 \mathrm{~ms}$ & $4 \mathrm{~ms}$ & $0.2 \mathrm{~ms}$ & $160 \mathrm{~ms}$ \\
\hline 4 & $0.19 \mathrm{~ms}$ & $0.01 \mathrm{~ms}$ & $4 \mathrm{~ms}$ & $0.2 \mathrm{~ms}$ & $160 \mathrm{~ms}$ \\
\hline
\end{tabular}

TABLE III

THE FITTED COEFFICIENTS FOR THE OPTIMAL TRANSFER FUNCTION

\begin{tabular}{|c|c|c|c|c|c|c|c|c|}
\hline $\mathrm{S}$ & $a_{0_{\mathrm{A}}}$ & $a_{1_{\mathrm{A}}}$ & $b_{1_{\mathrm{A}}}$ & $b_{2_{\mathrm{A}}}$ & $a_{0_{\mathrm{C}}}$ & $a_{1_{\mathrm{C}}}$ & $b_{1_{\mathrm{C}}}$ & $b_{2_{\mathrm{C}}}$ \\
\hline 1 & .042 & -.049 & -1.891 & .891 & -.011 & .011 & -1.999 & .999 \\
\hline 2 & .004 & -.006 & -1.946 & .946 & -.006 & .006 & -1.999 & .999 \\
\hline 3 & -.083 & .082 & -1.990 & .990 & -.007 & .007 & -1.999 & .999 \\
\hline 4 & -.084 & .083 & -1.992 & .992 & -.006 & .006 & -1.999 & .999 \\
\hline
\end{tabular}

\section{B. Predicting the WDR Neuron Spiking Activity}

A single point process model is able to efficiently provide an accurate estimation of the WDR neuron spiking activity. Comparing the ROC curves in Figure 5, we see that the full point process model (M3) can more accurately predict the WDR spiking activity when compared to the M1 (only LFP response) and M2 (only spiking history) models because it is farther away from the diagonal line representing chance. 


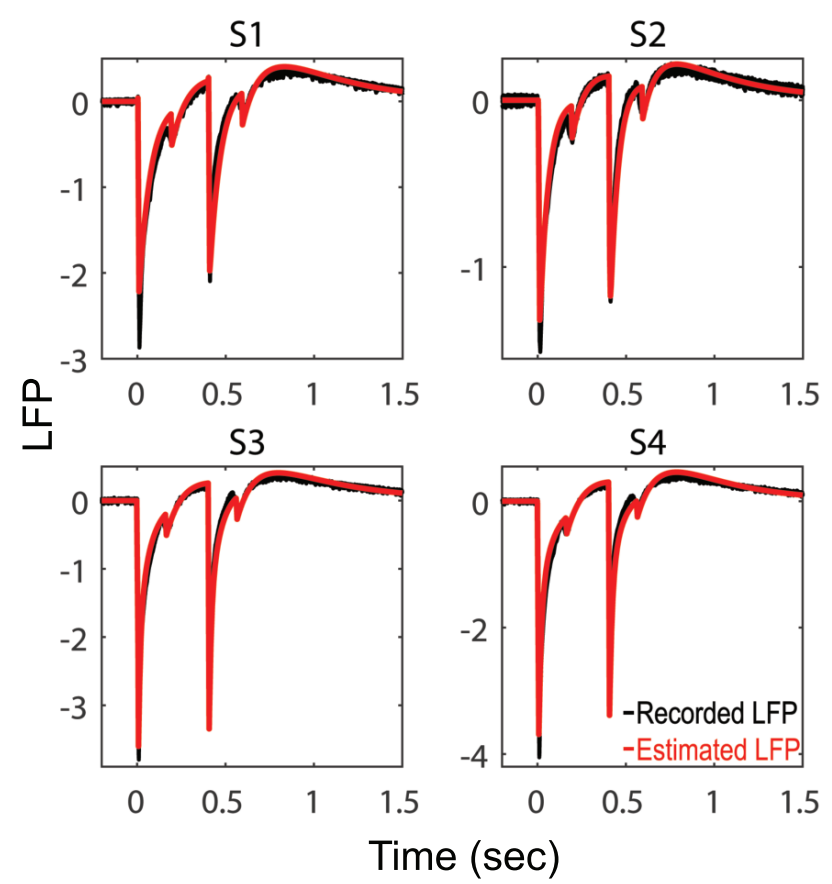

Fig. 4. A comparison of the recorded and estimated LFP responses from the optimized transfer function for each rat subject.


Fig. 5. The ROC curves for the three point process models of WDR activity. The thick line and shaded area indicate the mean and standard deviation over the 10 folds, respectively. The diagonal line represents chance.

To quantify the differences between the point process models, the AUC values from all ten folds are compared in a one-way ANOVA to determine if the model type is a significant factor. The results shown in Figure 6 indicate that the AUC values for the models are significantly different $(p<0.0001)$. The F-statistic value is shown for each respective ANOVA. Pairwise comparisons of the models show that the AUC values for the full model (M3) is significantly much higher than M1 $(p<0.0001)$, over all of the rats. For three of the four rats, M3 is also significantly higher than M2.

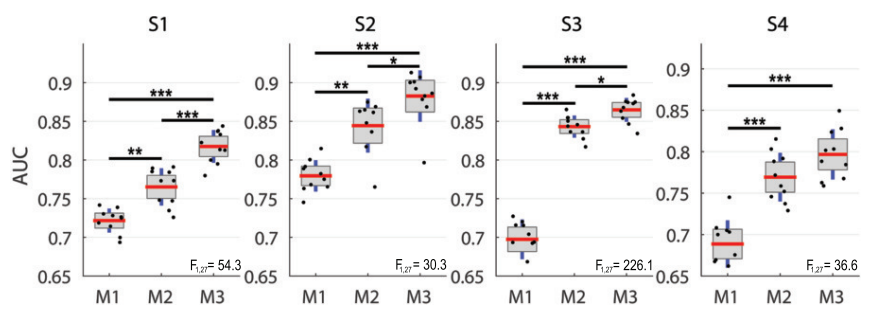

Fig. 6. A statistical comparison of the AUC values, from the ROC curves for all ten folds in Figure 5, for the three point process models. $* p<0.05$, $* * p<0.0005$, *** $p<0.0001$.

Therefore, in order to fully capture the neural dynamics of the WDR neurons then the input, LFP responses, and the spiking history must be taken into account.

\section{DISCUSSION}

In this paper, we construct a tractable computational model of the DH in healthy rats based on LFP and WDR recordings obtained from the superficial lamina network and the deep lamina, respectively. We can accurately predict responses of the DH circuit to electrical stimulation of the peripheral sciatic nerve by combining a deterministic LTI model with a stochastic point process model. The model is computationally efficient, low-dimensional, and able to capture the stochastic nature of neuronal dynamics in the DH. Future work includes estimating this model for the nerve-injured or $\mathrm{CP}$ animal, and then applying closed-loop stimulation strategies to steer the CP model to mimic a healthy model.

\section{REFERENCES}

[1] L. S. Simon, "Relieving pain in america: A blueprint for transforming prevention, care, education, and research," Journal of Pain \& Palliative Care Pharmacotherapy, vol. 26, no. 2, pp. 197-198, 2012.

[2] H. Breivik, B. Collett, V. Ventafridda, R. Cohen, and D. Gallacher, "Survey of chronic pain in europe: prevalence, impact on daily life, and treatment," European Journal of Pain, vol. 10, no. 4, pp. 287-333, 2006.

[3] R. Melzack, P. D. Wall, et al., "Pain mechanisms: a new theory," Science, vol. 150, no. 3699, pp. 971-979, 1965.

[4] Y. Le Franc and G. Le Masson, "Multiple firing patterns in deep dorsal horn neurons of the spinal cord: computational analysis of mechanisms and functional implications," Journal of neurophysiology, vol. 104, no. 4, pp. 1978-1996, 2010.

[5] P. C. Aguiar, M. M. Sousa, and D. Lima, "Nmda channels together with 1-type calcium currents and calcium activated non-specific cationic currents are sufficient to generate windup in wdr neurons." American Journal of Physiology-Heart and Circulatory Physiology, 2010.

[6] T. C. Zhang, J. J. Janik, and W. M. Grill, "Modeling effects of spinal cord stimulation on wide-dynamic range dorsal horn neurons: influence of stimulation frequency and gabaergic inhibition," Journal of neurophysiology, vol. 112, no. 3, pp. 552-567, 2014.

[7] D. R. Cox and V. Isham, Point processes. Routledge, 2018.

[8] D. Snyder and M. Miller, "Random point processes in space and time," Springer-Verlag, New York, NY, 1991.

[9] R. Q. Quiroga, Z. Nadasdy, and Y. Ben-Shaul, "Unsupervised spike detection and sorting with wavelets and superparamagnetic clustering," Neural Computation, vol. 16, no. 8, pp. 1661-1687, 2004.

[10] W. Truccolo, U. T. Eden, M. R. Fellows, J. P. Donoghue, and E. N. Brown, "A point process framework for relating neural spiking activity to spiking history, neural ensemble, and extrinsic covariate effects," Journal of Neurophysiology, vol. 93, no. 2, pp. 1074-1089, 2005.

[11] E. Brown, Methods and Models in Neurophysics. Elsevier, 2005, ch. 14 , pp. 691-726.

[12] E. N. Brown, R. Barbieri, U. T. Eden, and L. M. Frank, "Likelihood methods for neural spike train data analysis," Computational Neuroscience: A Comprehensive Approach, pp. 253-286, 2003.

[13] P. McCullagh, Generalized linear models. Routledge, 2018. 